\title{
Photoelectric Conversion Effect in Non-Photovoltaic Photorefractive Materials
}

\author{
Jaime Frejlich $^{1}$, Ivan de Oliveira ${ }^{2}$, Jesiel F. Carvalho ${ }^{3}$, William R. Araújo ${ }^{2}$, Marc Georges ${ }^{4}$ and Thizy \\ Cedric $^{4}$ \\ 1-Universidade Estadual de Campinas, Instituto de Física IFGW, Campinas-SP, Brazil \\ 2-Universidade Estadual de Campinas, Faculdade de Tecnologia FT, Limeira-SP, Brazil \\ 3-Universidade Federal de Goiás, Departamento de Física, Goiânia-GO, Brazil \\ 4-Centre Aerospatiale de Liège, Liège, Belgique \\ frejlich@ifi.unicamp.br
}

\begin{abstract}
We report on the photoelectric conversion at a non-photovoltaic photorefractive material sandwiched between transparent conductive electrodes. Its behavior is based on the light-induced Schottky effect. Its nature and performance was clearly established by wavelength-resolved photoconductivity.

OCIS codes: Photoconductivity (040.5150); Photorefractive Materials (160.5320); Optoelectronics (230.0250)
\end{abstract}

\section{Introduction}

Light-induced Schottky effect at a transparent conductive electrode-bulk photorefractive crystal interface was already shown [1] to be due to the large density of electron-filled localized states in most photorefractive materials [2] that allow to produce a large density of free electrons in the conduction band (CB), close to the illuminated transparent conductive electrode., by the action of light of adequate wavelength. Free electrons in the CB diffuse to the electrode until a sufficiently large depletion layer and associated electric barrier is build up to stabilize the process. The same barrier but of opposite polarization is build up at the rear photorefractive-electrode interface. As light is strongly absorbed while going through the photorefractive slice, the electric potential barrier is much weaker at the less illuminated rear interface than at the more illuminated front one and such an unbalanced effect produces an overall transport of photoelectrons, generated in the front depletion layer, through the sandwiched photorefractive.

\section{Results}

Preliminary measurements carried on a $0.8 \mathrm{~mm}$ thick slice of photorefractive Bismuth Titanate $\left(\mathrm{Bi}_{12} \mathrm{TiO}_{20}\right)$ [3] sandwiched between ITO electrodes with an input illuminated surface of about $50 \mathrm{~mm}^{2}$ have shown that the overall light-induced photocurrent increases as illumination wavelength decreases in agreement with light-absorption coefficient known to increase with decreasing wavelength producing a desirable larger unbalanced front-to-rear electric potential barrier. From wavelength-resolved photoconductivity data, using rather low light irradiances, we estimated an overall $0.4 \mu \mathrm{A}$ photocurrent produced by $50 \mathrm{~mW} @ 2.5 \mathrm{eV}$ (approx $\lambda=500 \mathrm{~nm}$ ) illumination onto the front electrode device surface. Measurements using higher light irradiances systematically gave much poorer photoelectric conversion efficiencies, probably because of the well known photochromic effect of $\mathrm{Bi}_{12} \mathrm{TiO}_{20}$ that is enhanced at higher irradiances. Although the present photoelectric conversion efficiency is rather low, its nature is clearly demonstrated and its performance may certainly be optimized by adequately selecting the photorefractive material and the slice's thickness in the device.

\section{References}

[1] Jaime Frejlich, Christophe Longeaud and Jesiel F. Carvalho, "Photoinduced Schottky Barrier in Photorefractive Materials", Phys.Rev.Lett. 104, 116601_1-16601_4 (2010)

[2] Jaime Frejlich, Photorefractive Materials (Wiley \& Sons, 2007)

[3] J.F.Carvalho and A.C. Hernandes, " $\mathrm{Bi}_{12} \mathrm{TiO}_{20}$-Single Crystals: A Study of Intrinsic Defects and Growth Parameters", J.Crys.Growth, 205, 185-190 (1999\} 